Supporting Information for

\title{
A light activated glucagon trimer with resistance to fibrillation
}

\author{
Swetha Chintala ${ }^{1}$ and Simon H. Friedman ${ }^{1 *}$
}

\begin{abstract}
${ }^{1}$ Division of Pharmacology and Pharmaceutical Sciences
School of Pharmacy, University of Missouri-Kansas City, Kansas City, MO 64108
\end{abstract}

*Telephone: 816-235-2224

Fax: 816-235-5779

Email: friedmans@umkc.edu

SI Pages S1-S25, SI Figures S1-S17 


\section{TABLE OF CONTENTS}

1 MATERIALS

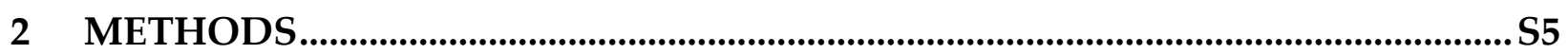

2.1 Synthesis of compound 6 (Glucagon mono-azide)................................................. S5

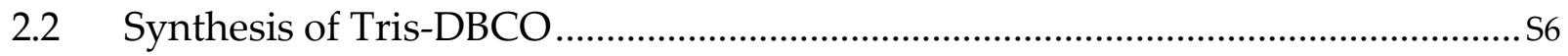

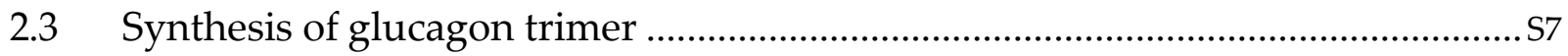

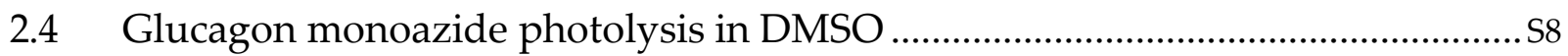

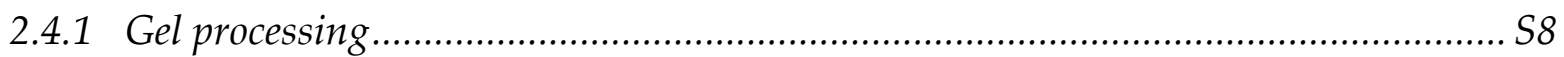

$2.5 \quad$ Isoelectric point of glucagon mono-azide ............................................................ 58

2.6 Glucagon trimer photolysis, supernatant analysis of centrifuged samples..........S8

2.7 Glucagon trimer photolysis, supernatant analysis of centrifuged samples with extended extraction ............................................................................................................ 9

2.8 Glucagon trimer photolysis, whole reaction analysis of suspended samples ... S10

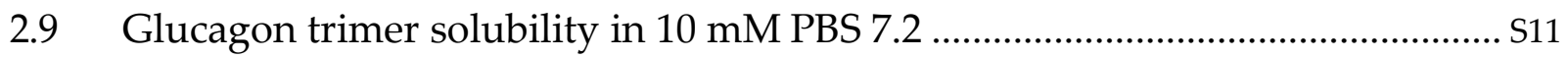

2.10 Glucagon trimer fibrillation studies .................................................................. S12

2.10.1 Sample preparation for thioflavin $T$ and turbidity assays ................................ S12

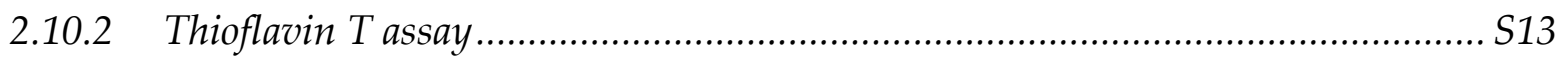

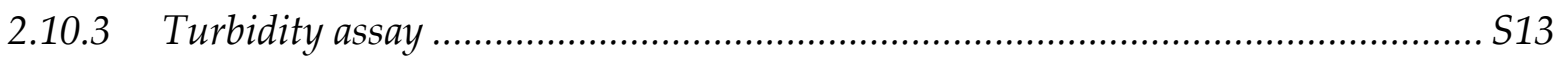

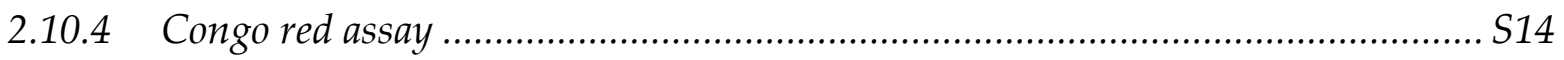

2.10.5 Particle size analysis using dynamic light scattering (DLS): ............................ S14

3 ANALYTICAL RESULTS.............................................................................................

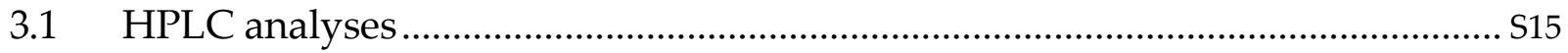

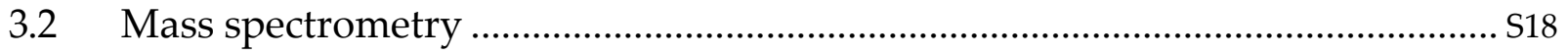

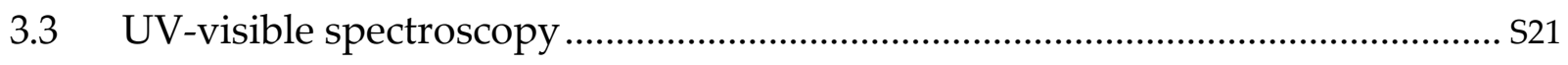

3.4 Isoelectric focusing of glucagon and glucagon mono-azide ............................... S23

3.5 Glucagon trimer photolysis, supernatant analysis of centrifuged samples with

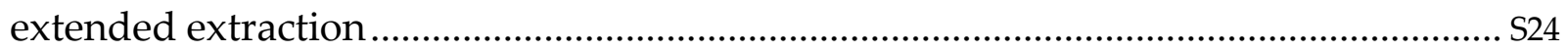

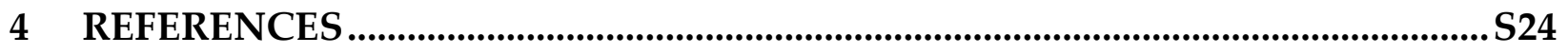




\section{MATERIALS}

Chemicals and supplies including acetonitrile, HPLC grade water, dimethyl sulfoxide, methanol, N-Methyl pyrrolidinone, ethyl acetate, methylene chloride, N, Ndimethylformamide, acetone, o-phosphoric acid, glacial acetic acid, diisopropylethylamine, sodium chloride, anhydrous magnesium sulfate, hydrochloric acid, sodium bicarbonate, sodium hydroxide, glutaraldehyde, isopropanol, ethyl alcohol, tert-butyl bromoacetate, trifluoroacetic acid, acetic anhydride, potassium carbonate, Coomassie Brilliant Blue R-250, Crocein Scarlet 3B, GIBCO phosphate buffered saline solution $\mathrm{pH}$ 7.4, Acros Organics ${ }^{\mathrm{TM}}$ silica gel for column chromatography (Ultra-Pure, 40-60 $\mu, 60 \AA$ ), Fisherbrand ${ }^{\mathrm{TM}}$ shell vials for Waters ${ }^{\mathrm{TM}}$ autosamplers, 1 kDA cut off dialysis membrane were purchased from Fisher Scientific. Other synthetic precursors and reagents including acetovanillone, 1,3,5-cy>clohexane carboxylic acid, 11-azido-3,6,9-trioxaundecan-1-amine, hydrazine monohydrate, manganese dioxide, thioflavin T, Congo red were purchased from Sigma Aldrich. HATU was purchased from ChemImpex. DBCO-amine was purchased from Click Chemistry tools. $\mathrm{pH}$ 3-10 Criterion ${ }^{\text {TM }}$ IEF gels (18 well), 10X IEF cathode buffer, 10X IEF anode buffer, IEF sample buffer, IEF standards, Criterion ${ }^{\mathrm{TM}}$ Cell gel running unit were purchased from Bio-Rad. Human glucagon was purchased from AmbioPharm. Other commercial sources of glucagon showed signs of pre-fibrillation and lower reactivity during synthesis. Yarra ${ }^{\mathrm{TM}}$ $3 \mu \mathrm{m}$ SEC-2000 and SEC column standards were purchased from Phenomenex. Black, clear bottom 96 well microplates used for thioflavin T assays and transparent 96 well microplates used for turbidity assays were purchased from Greiner.

An Ocean Optics USB-2000 fiber optic spectrophotometer with DT-Mini-B lamp source was used for UV/visible spectrophotometric analysis. Analytical HPLC analysis was performed on an Agilent 1050 HPLC with a diode array detector, using a 150 X $3.2 \mathrm{~mm}$ $5 \mu \mathrm{m}$ Nucleosil $\mathrm{C}_{18}$ column (Supelco Analytical from Sigma Aldrich). Mass spectrometry was performed using a Q-Trap 3200 (electrospray ionization) attached to a Shimadzu UFLC system. Analyst v.1.4.2 software was used for the LC-MS analyses. Masses greater than 1700 were obtained by deconvoluting primary spectra of the different charge states using the Bioanalyst software. Preparative purification of compounds was done on a Thermo Scientific Ultimate 3000 HPLC with an automated fraction collector, using a Phenomenex 250 X $21.2 \mathrm{~mm} 10 \mu \mathrm{m} \mathrm{C} 18$ column. Micro pH NMR glass electrode from Thermo Scientific Orion connected to an Orion $\mathrm{pH}$ meter was 
used to determine and adjust $\mathrm{pH}$ of smaller volume samples. A compact light source using a Nichia $365 \mathrm{~nm}$ LED was used for photolysis studies as previously described [1]. Composition of PBS used for various experiments:

1) PBS used for photolysis and solubility experiments: $10 \mathrm{mM}$ Phosphate Buffer Saline with $150 \mathrm{mM} \mathrm{NaCl}$, pH 7.2

2) PBS used for fibrillation kinetics assay: $10 \mathrm{mM}$ Phosphate Buffer Saline with 150 $\mathrm{mM} \mathrm{NaCl}, \mathrm{pH} 2.5$

3) PBS used for preparing Congo red dye stock and working solution: $5 \mathrm{mM}$ Phosphate Buffer Saline with $150 \mathrm{mM} \mathrm{NaCl}$, pH 7.4

4) PB used for dialysis of purified glucagon HPLC fractions: $100 \mathrm{mM}$ Phosphate buffer (no saline), $\mathrm{pH} 7$ 


\section{METHODS}
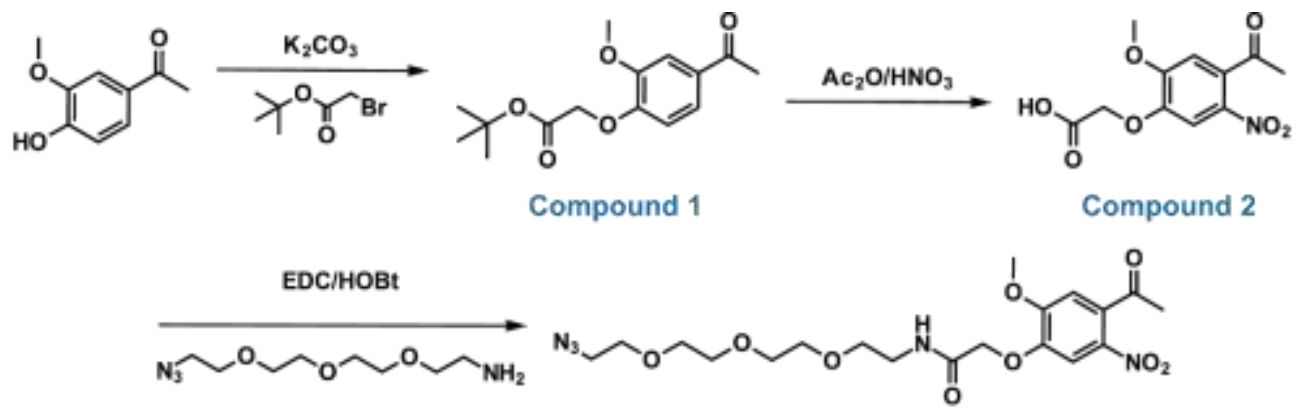

Compound 3

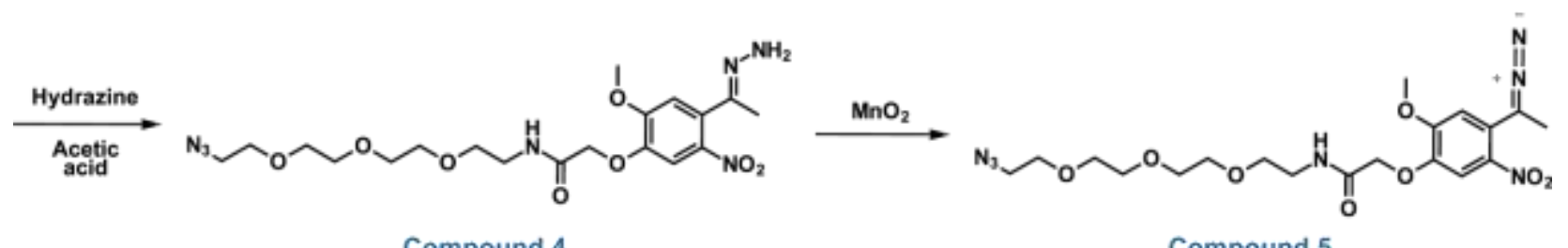

Compound 4

Compound 5

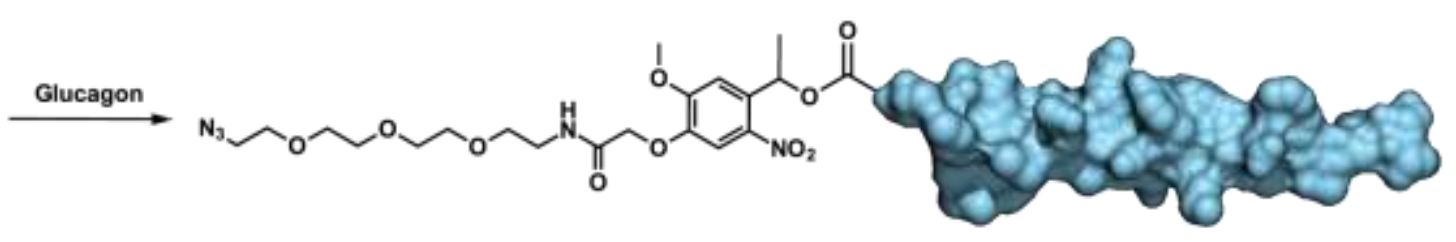

Compound 6

Compound 1 and 2 were synthesized as described by Holmes et al [2]. Compounds 3 and 4 were synthesized as discussed by Jain et al [1].

\section{Synthesis of compound 6 (Glucagon mono-azide)}

$89.8 \mu$ moles of compound 4 (hydrazone) was dissolved in DMSO at $0.1 \mathrm{M}$ and combined with 2.7 mmoles $\left(230 \mathrm{mg}\right.$ ) of $\mathrm{MnO}_{2}$. The reaction was shaken vigorously for 45 minutes. After 45 minutes, the reaction was centrifuged at $20800 \mathrm{~g}$ for 10 minutes. Compound 5 was not isolated and characterized. The supernatant containing diazo was added to a freshly prepared solution of glucagon $(28.7 \mu$ moles $(100 \mathrm{mg})$ of glucagon in 8 $\mathrm{mL}$ DMSO). Additional DMSO was added to make up the volume of the reaction 14.3 $\mathrm{mL}$ and adjust the final concentration of glucagon to $2 \mathrm{mM}$. The reaction mixture was incubated protected from light at room temperature for 16 hours. The reaction was analyzed for the formation of the product using reversed phase HPLC. Reversed phase HPLC (flow rate $0.4 \mathrm{~mL} / \mathrm{min}$, runtime: 30 minutes with 5 minutes post-run), solvent $\mathrm{A}$ (0.1\% TFA in $\mathrm{H} 2 \mathrm{O})$, solvent $\mathrm{B}(0.1 \%$ TFA in acetonitrile), gradient $0 \% \mathrm{~B}$ to $100 \% \mathrm{~B}$ over 28 minutes, $100 \%$ B to $0 \%$ B over 2 minutes, post-run $0 \%$ B for 5 minutes, $\mathrm{C}_{18}$ Supelco 
Nucleosil column $(5 \mu \mathrm{m}, 150 \times 3.2 \mathrm{~mm})$. Glucagon monoazide (GMA) eluted at 19 minutes. Glucagon mono-azide was purified using semi preparative reversed phase HPLC. Reversed phase HPLC (flow rate $2 \mathrm{~mL} / \mathrm{min}$, runtime: 65 minutes with 10 minutes pre-run), solvent $\mathrm{A}\left(0.1 \%\right.$ TFA in $\left.\mathrm{H}_{2} \mathrm{O}\right)$, solvent $\mathrm{B}(0.1 \%$ TFA in acetonitrile), gradient $0 \%$ B to $60 \%$ B over 45 minutes, gradient $60 \%$ B to $100 \%$ B over 15 minutes, prerun $0 \% \mathrm{~B}$ for 10 minutes, $\mathrm{C}_{18}$ Phenomenex Luna column $(5 \mu \mathrm{m}, 250 \times 10 \mathrm{~mm})$. Masses of glucagon, glucagon mono-azide, glucagon di-azide was confirmed by the direct infusion of respective fractions in ESI-MS in 50\% v/v $\mathrm{H}_{2} \mathrm{O} / \mathrm{ACN}(0.1 \%$ TFA) See manuscript figure 2a). ESI-MS (m/z): [M] calculated for glucagon, 3485.0; observed deconvoluted mass, 3483.0; [M] calculated for glucagon mono-azide, 3938.2; observed deconvoluted mass, 3938.0; [M] calculated for glucagon di-azide, 4391.4; observed deconvoluted mass, 4392.0; UV/vis (GMA, DMSO): $\lambda \max : 284 \mathrm{~nm},\left(\varepsilon 346 \mathrm{~nm}: 4470 \mathrm{M}^{-1}\right.$ $\mathrm{cm}^{-1}$ ). See Figure S1, Figure S6 and Figure S12 in the Supporting Information for HPLC, MS and UV-visible spectroscopic analyses of GMA.

\section{Synthesis of Tris-DBCO}

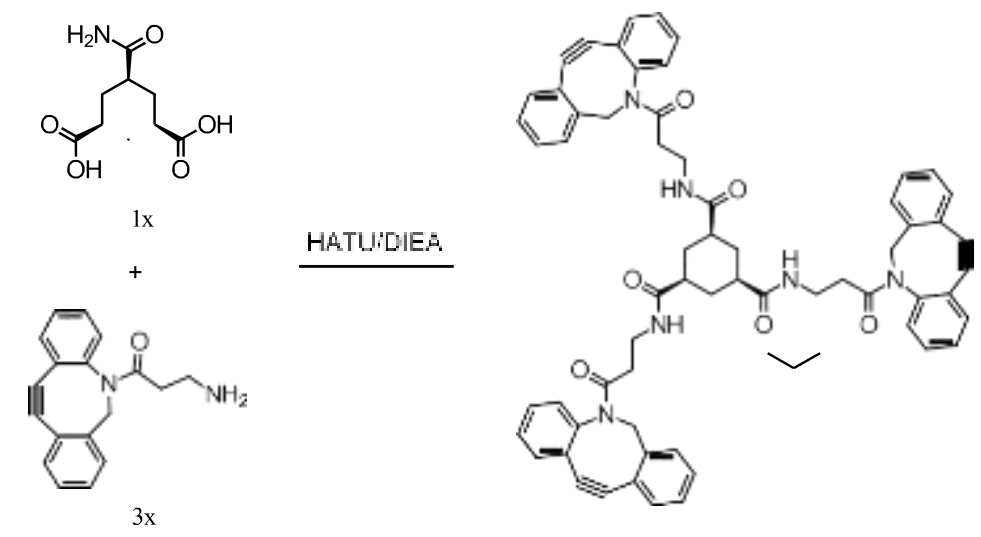

The synthesis was adapted from Sarode et al. [3]. 619 umoles (235.4 mg) of HATU was added to $103 \mu$ moles $(22.3 \mathrm{mg}$ ) of 1,3,5, cyclohexane tricarboxylic acid in DMF. To this mixture, $619 \mu$ moles $(107.8 \mu \mathrm{L})$ of DIEA was added. Finally, $619 \mu$ moles $(171 \mathrm{mg})$ of DBCO-amine was added to the activated tricarboxylic acid. The total reaction volume was then made up to $5.15 \mathrm{~mL}$ with NMP. The reaction proceeded for 2 hours at room temperature. The product formation was confirmed using reversed phase HPLC. Reversed phase HPLC (flow rate $0.4 \mathrm{~mL} / \mathrm{min}$, run time: 30 minutes with 5 minutes postrun), solvent $\mathrm{A}$ (water), solvent $\mathrm{B}$ (acetonitrile), gradient $0 \% \mathrm{~B}$ to $100 \%$ B over 25 minutes, $100 \%$ B from 25 to 30 minutes, post-run $0 \%$ B for 5 minutes, $\mathrm{C}_{18}$ Supelco analytical Nucleosil column $(5 \mu \mathrm{m}, 150 \times 3.2 \mathrm{~mm})$. The product eluted at 26.6 minutes. 
Tris-DBCO was purified using semi preparative reversed phase HPLC. Reversed phase HPLC (flow rate $2 \mathrm{~mL} / \mathrm{min}$, runtime: 45 minutes with 10 minutes pre-run), solvent $\mathrm{A}$ (water), solvent B (acetonitrile), gradient $0 \%$ B to $100 \%$ B over 35 minutes, $100 \%$ B from 35 to 45 minutes, pre-run $0 \%$ B for 10 minutes, $C_{18}$ Phenomenex Luna column $(5 \mu \mathrm{m}, 250$ x 10mm). Tris-DBCO product was collected from 40.8 minutes to 41.15 minutes. Trifluoroacetic acid was not used in HPLC solvents to avoid DBCO degradation in acidic conditions. Identity of tris-DBCO was determined using direct infusion ESI-MS in $100 \%$ ACN. ESI-MS (m/z): $[\mathrm{M}+\mathrm{H}]^{+}$calculated for $\mathrm{C}_{63} \mathrm{H}_{54} \mathrm{~N}_{6} \mathrm{O}_{6}$, 991.410; observed, 991.4; UV/vis (DBCO-amine, DMSO): $\lambda \max : 291 \mathrm{~nm}\left(\varepsilon 312 \mathrm{~nm}: 34500 \mathrm{M}^{-1} \mathrm{~cm}^{-1}\right)$ [3]. See Figure S2, Figure S7 and Figure S13 in the Supporting Information for HPLC, MS and UVvisible spectroscopic analyses.

\section{Synthesis of glucagon trimer}

$3.18 \mu$ moles of glucagon mono-azide was combined with $0.64 \mu$ moles of Tris-DBCO. Purified and lyophilized glucagon monoazide and tris-DBCO were reconstituted in DMSO and quantitated using UV/vis spectrophotometer. The reaction volume was adjusted to $1.5 \mathrm{~mL}$ with DMSO and kept at room temperature overnight. Although the reaction rates were high at higher reactant concentrations, we used relatively low concentration to prevent glucagon aggregation at higher concentrations.

The product formation was confirmed by size exclusion chromatography. Size exclusion HPLC (flow rate $1 \mathrm{~mL} / \mathrm{min}$, run time: 20 minutes), solvent $\mathrm{A}$ (water), solvent B ( $0.1 \%$ TFA in acetonitrile), $55 \%$ solvent A, $45 \%$ solvent $B$, isocratic mobile phase, Phenomenex Yarra $3 \mu \mathrm{m}$ SEC-2000 size exclusion column $(3 \mu \mathrm{m}, 300 \times 7.8 \mathrm{~mm})$. The glucagon trimer product eluted at 5.4 minutes. The product was purified using size exclusion chromatography. SEC (flow rate $0.5 \mathrm{~mL} / \mathrm{min}$, runtime: 30 minutes), solvent A (water), solvent $\mathrm{B}(0.1 \%$ TFA in $\mathrm{ACN}), 55 \%$ solvent $\mathrm{A}, 45 \%$ solvent $\mathrm{B}$, isocratic mobile phase, Phenomenex Yarra $3 \mu \mathrm{m}$ SEC-2000 size exclusion column $(3 \mu \mathrm{m}, 300 \times 7.8 \mathrm{~mm})$. The trimer product was collected from 10.7-11.4 minutes. Identity of glucagon trimer was determined using direct infusion ESI-MS in 50\% v/v $\mathrm{H}_{2} \mathrm{O} / \mathrm{ACN}(0.1 \% \mathrm{TFA})$. ESI-MS $(\mathrm{m} / \mathrm{z})$ : $[\mathrm{M}]$ calculated for glucagon trimer, 12805.0; observed deconvoluted mass, 12805.0; UV/vis (glucagon trimer, DMSO): $\lambda \max : 284 \mathrm{~nm}$, $\left(\varepsilon 346 \mathrm{~nm}: 13410 \mathrm{M}^{-1} \mathrm{~cm}^{-1}\right)$. See Figure S3, Figure S8 and Figure S14 in the Supporting Information for HPLC, MS and UV-visible spectroscopic analyses. 


\section{Glucagon monoazide photolysis in DMSO}

40 nmoles of glucagon monoazide were combined with $40 \mu \mathrm{L}$ of DMSO. This solution was then irradiated with a $365 \mathrm{~nm}$ LED light source at a distance of $7 \mathrm{~cm}$ (absolute irradiance $=934 \mu \mathrm{Watt} / \mathrm{cm}^{2}$ ) for $1,2,3,4,5,10,15,30,60$ minutes. $3 \mu \mathrm{L}$ of photolysis solution was collected after each irradiation. Collected samples were diluted with $7 \mu \mathrm{L}$ of IEF sample buffer and loaded into the wells of a $\mathrm{pH} 3-10$ Criterion $^{\mathrm{TM}}$ IEF gel. IEF gel electrophoresis was performed following the protocol from [5]. The photolysis mixture was also analyzed using $\mathrm{C}_{18}$ HPLC chromatography. The photoreleased glucagon was quantitated using a HPLC glucagon standard curve and the cumulative glucagon release was plotted against irradiation time and fit using first order rate law.

\subsubsection{Gel processing}

After IEF gel runs, gels were fixed for 2 hours using a fixing solution (30\% glutaraldehyde in $30 \%$ methanol). The gel was then washed with a washing solution (27\% isopropanol, $10 \%$ acetic acid) for 5 minutes and stained for 45 minutes with $0.1 \mathrm{X}$ staining solution (1X staining solution: $0.04 \% \mathrm{w} / \mathrm{v}$ Coomassie Blue R-250, 0.05\% w/v Crocein Scarlet in 27\% isopropanol, $10 \%$ acetic acid). The gel was then destained until the bands were clearly visible. First wash was performed using $20 \%$ methanol, $5 \%$ acetic acid solution for 120 minutes. Successive washes were performed using $40 \%$ methanol, $10 \%$ acetic acid solution until the gel background becomes clear. Overnight destaining was performed using $20 \%$ methanol, $5 \%$ acetic acid.

\section{Isoelectric point of glucagon mono-azide}

The isoelectric point was determined as described earlier [5]. Glucagon standard, glucagon mono-azide and IEF standards were loaded into wells of IEF gel. Gel was run and processed as described in Section 2.4. The line segment tool in Adobe Illustrator was used to measure peptide migration distance from bottom of well to the peptide band. A standard curve was constructed using the migration distances of the IEF standards and their isoelectric points. The slope of the fit of this data was used to determine the $\mathrm{pI}$ of glucagon and glucagon mono-azide. For the IEF gel and the corresponding standard plot, see Figure S15 and Figure S16.

\section{Glucagon trimer photolysis, supernatant analysis of centrifuged samples}

The purified glucagon trimer was dissolved in DMSO and quantified using UV spectroscopy $\left(\varepsilon 346=13410 \mathrm{M}^{-1} \mathrm{~cm}^{-1}\right)$. A solution containing $33.3 \mathrm{nmol}$ of glucagon trimer 
was transferred into each of two Eppendorf tubes (test and control) and dried in vacuum. $100 \mu \mathrm{L}$ of $10 \mathrm{mM}$ PBS 7.2 was added to each of the test and control tubes and the $\mathrm{pH}$ was adjusted to 7.2 with $0.1 \mathrm{~N} \mathrm{NaOH}$. The trimer suspensions were then homogenized using a battery-operated homogenizer for 4 minutes as previously described [4]. The trimer suspension in the test tube was irradiated with a $365 \mathrm{~nm}$ LED light source (absolute irradiance $=934 \mu \mathrm{Watt} / \mathrm{cm}^{2}$ ) at a distance of $7 \mathrm{~cm}$, while the control tube was shielded from light. Both the test and control suspensions were treated identically for analysis. Briefly, suspensions were vortexed for 45 seconds, and then centrifuged for five minutes and the entire $100 \mu \mathrm{L}$ of the clarified supernatants were collected. The remaining trimer pellets in both the test and control tubes were then resuspended in fresh $100 \mu \mathrm{L}$ buffer and the next irradiation period was initiated. $30 \mu \mathrm{L}$ of each of the samples were analyzed using reversed phase chromatography on a $\mathrm{C}_{18}$ column. Photoreleased glucagon in each sample was determined using glucagon standards of known concentration. The photoreleased glucagon (cumulative concentration determined by dividing cumulative moles by the $100 \mu$ photolysis volume) was plotted as a function of time to study the rate of photolysis. Data was fitted using the following first order equation:

$\mathrm{A}=\mathrm{A}_{0}\left(1-\mathrm{e}^{-\mathrm{kt}}\right)$

$\mathrm{A}_{0}=$ glucagon trimer concentration at $\mathrm{t}=0$ minutes

\section{Glucagon trimer photolysis, supernatant analysis of centrifuged samples with extended extraction}

A suspension of lyophilized glucagon trimer (33.3 nmol in $100 \mu \mathrm{L}$ of $10 \mathrm{mM}$ PBS 7.2) was briefly homogenized with a battery-operated tissue homogenizer. The $\mathrm{pH}$ of the suspension was adjusted to 7.2 with $0.1 \mathrm{~N}$ sodium hydroxide. This suspension was then further homogenized for 4 minutes as described earlier [4]. The glucagon trimer was photolyzed with our $365 \mathrm{~nm}$ light source at a distance of $7 \mathrm{~cm}$. Irradiation of the material was performed for a duration of 60 minutes. Irradiation was interrupted to collect samples at 1, 2, 3, 4, 5, 10, 15, 30 and 60 minutes. For sampling, the suspension was first vortexed for 45 seconds, centrifuged for 5 minutes and the entire clarified supernatant was collected for glucagon analysis. Prior to the next irradiation step, the pellet was resuspended in $100 \mu \mathrm{L}$ of fresh buffer, vortexed for 5 minutes and centrifuged for 5 minutes to extract undissolved photoreleased glucagon. This was repeated one more time $(100 \mu \mathrm{l}$ fresh buffer, 5 minutes of vortexing, 5 minutes of 
centrifugation) for a total of three washings. $30 \mu \mathrm{L}$ of each sampled supernatant was injected on HPLC to quantitate the amount of glucagon released. A glucagon standard curve was run to correlate peak integration with amount. The cumulative concentration of glucagon released (determined by dividing cumulative released moles by the $100 \mu \mathrm{l}$ photolysis volume) was plotted against time and fitted to a first order equation in Kaleidagraph as described above in section 2.6. (See figure S17 in Supporting Information for the graph)

\section{Glucagon trimer photolysis, whole reaction analysis of suspended samples}

$1.41 \mathrm{mg}$ of lyophilized glucagon trimer was combined with $250 \mu \mathrm{L}$ of $10 \mathrm{mM}$ PBS 7.2. The material was soluble, and the $\mathrm{pH}$ of the solution was found to be acidic due to the presence of TFA salts carried over from HPLC solvents used for trimer purification. The $\mathrm{pH}$ was then adjusted to 7.2 using $0.1 \mathrm{~N} \mathrm{NaOH}$ and $0.1 \mathrm{~N} \mathrm{HCl}$, and the material precipitated as a white suspension. The trimer suspension was then homogenized using a battery operated homogenizer for 4 minutes [4]. A small amount of the trimer suspension was sampled and diluted in DMSO to determine the concentration using UV spectroscopy $\left(\varepsilon 346=13410 \mathrm{M}^{-1} \mathrm{~cm}^{-1}\right)$. The concentration of the trimer suspension was then adjusted to $0.33 \mathrm{mM}$ (equivalent to $1 \mathrm{mM}$ glucagon) using PBS 7.2. The glucagon trimer suspension was then distributed equally in volume into two $1 \mathrm{~mL}$ glass vials (50 nmoles of trimer per vial). The material in one vial was irradiated and the other vial was shielded from light as a control. The trimer in the irradiation vial was irradiated with the $365 \mathrm{~nm}$ LED light source at a distance of $7 \mathrm{~cm}$ for 1, 2, 3, 4, 5, 10, 15, 30, 60 minutes. Absolute irradiance of the $365 \mathrm{~nm}$ LED at $7 \mathrm{~cm}$ was found to be 934 $\mu W a t t / \mathrm{cm}^{2}$. After each irradiation, the reaction mixture was suspended well using a pipette, $4 \mu \mathrm{L}$ of the suspension was immediately sampled and diluted with $11 \mu \mathrm{L}$ of DMSO to make a completely solubilized solution. $10 \mu \mathrm{L}$ of this sample was analyzed using size exclusion HPLC. An overlay of size exclusion HPLC analysis of photolysis mixture after irradiation is included in this Supporting Information (Figure S4). The known concentration of the trimer at time zero and its corresponding HPLC absorbance integration value determined at $345 \mathrm{~nm}$ was used as single point standard to calculate concentrations of trimer, dimer and monomer from integration at the remaining time points. Note that the molar absorbance for all the species of trimer, dimer and monomer at $345 \mathrm{~nm}$ is the same, as they have the common tris-DBCO central moiety linked to three DMNPE groups. Based on the concentration of trimer, dimer and monomer in the photolysis mixture, the photoreleased glucagon concentration was determined at 60 
minutes. This is used as a single point standard to determine the concentration of photoreleased glucagon at the remaining time points, using its corresponding HPLC integration units at $280 \mathrm{~nm}$. Finally, the photoreleased glucagon concentration was corrected by subtracting the monomer concentration at each time point, as the monomer co-eluted with glucagon and contributed to the overall $280 \mathrm{~nm}$ absorbance. Note that the molar absorbance of monomer and glucagon at $280 \mathrm{~nm}$ is marginally different, resulting in small error in photoreleased glucagon concentration. The error was predicted to be negligible considering the low monomer concentration compared to photoreleased glucagon at all time points. Glucagon trimer, dimer and the photoreleased glucagon were collected during the SEC analytical runs and characterized by mass spectrometry (Figure S9, Figure S10 and Figure S11). The concentration of each species were plotted against irradiation time. The trimer and photoreleased glucagon curves were fitted with first order rate equations using Kaleidagraph.

Equation for fitting glucagon trimer: $\mathrm{A}=\mathrm{A}_{0} \cdot \mathrm{e}^{-\mathrm{kt}}$

$\mathrm{A}_{0}=$ glucagon trimer concentration at $\mathrm{t}=0$ minutes

Equation for fitting photoreleased glucagon: $\mathrm{B}=\mathrm{A}_{0}\left(1-\mathrm{e}^{-\mathrm{kt}}\right)$

$\mathrm{A}_{0}=$ glucagon trimer concentration at $\mathrm{t}=0$ minutes

\subsection{Glucagon trimer solubility in $10 \mathrm{mM}$ PBS 7.2}

The solubility of the glucagon trimer was compared to the solubility of native glucagon in $10 \mathrm{mM}$ PBS at $\mathrm{pH}$ 7.2. Glucagon standard and glucagon trimer samples were performed in triplicates. $5.04 \mathrm{mg}(1.4 \mu$ moles $)$ of glucagon standard was weighed into a $1.8 \mathrm{~mL}$ Eppendorf tube and combined with $200 \mu \mathrm{L}$ of $10 \mathrm{mM}$ PBS 7.2. The glucagon trimer was purified using SEC as described in Section 2.3. Purified glucagon trimer HPLC fractions were dialyzed against $100 \mathrm{mM}$ phosphate buffer followed by overnight desalting. Water was changed once during overnight desalting. The glucagon trimer precipitated in this process. Trimer suspension was kept at $-80{ }^{\circ} \mathrm{C}$ until lyophilization. 4 $\mathrm{mg}$ (312 nmoles) of purified and lyophilized glucagon trimer was weighed into a $1.8 \mathrm{~mL}$ Eppendorf tube and combined with $200 \mu \mathrm{L}$ of $10 \mathrm{mM}$ PBS 7.2. The $\mathrm{pH}$ of both glucagon standard and glucagon trimer samples were adjusted to $\mathrm{pH} 7.2$ using $0.1 \mathrm{~N} \mathrm{NaOH}$. Both the samples were homogenized for 4 minutes using a battery-operated homogenizer. The glucagon samples were vortexed gently for 2 hours to allow the samples to reach saturation. Samples were turbid indicating that samples reached the solubility limit. 
These tubes were then centrifuged at $20000 \mathrm{~g}$ for 15 minutes to pellet the insoluble material. The supernatant from the centrifuge samples were collected into fresh Eppendorf tubes after separating it from the insoluble pellet. Supernatant samples were analyzed using HPLC. Concentration of glucagon and glucagon trimer in the supernatant were estimated using glucagon and glucagon trimer HPLC standard curves.

\section{Glucagon trimer fibrillation studies}

The fibrillation kinetics of the glucagon trimer were assayed using thioflavin $\mathrm{T}$ fluorescence and turbidity as described previously [6]. In this study, fibrillation of the lyophilized glucagon trimer was compared with a glucagon control. The glucagon control was prepared by processing the commercially obtained glucagon using identical conditions to those experienced during the synthesis of the glucagon trimer. The glucagon control was prepared in the following way: glucagon was dissolved in DMSO at $2 \mathrm{mM}$ concentration simulating the glucagon-diazo reaction overnight at room temperature. This glucagon was then mock purified using the same glucagon monoazide purification method described in Section 2.1. Combined multiple vials of HPLC fractions were transferred to a $1 \mathrm{kDA}$ cut off dialysis membrane and dialyzed against $100 \mathrm{mM}$ phosphate buffer (not Saline) pH 7 for 1 hour, followed by overnight desalting in deionized water. Water was changed once in the desalting process. The dialyzed HPLC fraction was emptied into multiple $20 \mathrm{ml}$ scintillation vials $(5 \mathrm{ml}$ per vial) and maintained at $-80{ }^{\circ} \mathrm{C}$ for $24-48$ hours and then lyophilized. The lyophilized glucagon was then dissolved in DMSO at $2.12 \mathrm{mM}$ and stored at room temperature overnight, mimicking glucagon trimer synthesis. This glucagon was mock purified using the trimer purification method described in Section 2.3. Combined HPLC fractions were dialyzed as described above and then lyophilized.

\subsubsection{Sample preparation for thioflavin $\mathrm{T}$ and turbidity assays}

$4.3 \mathrm{mg}$ of glucagon trimer and $4.78 \mathrm{mg}$ of glucagon control were weighed, and both the samples were dissolved in $10 \mathrm{mM}$ PBS with $150 \mathrm{mM} \mathrm{NaCl}, \mathrm{pH} 2.5$ at concentrations of 95.6 $\mu \mathrm{M}$ and $286.9 \mu \mathrm{M}$ respectively. The $\mathrm{pH}$ of these solutions was checked and adjusted to 2.5 using $0.1 \mathrm{~N} \mathrm{NaOH}$ and $0.1 \mathrm{~N} \mathrm{HCl}$. Both the solutions were filtered through $0.22 \mu \mathrm{m}$ filter. The filtered samples were centrifuged at $20000 \mathrm{~g}$ for 5 minutes and the supernatant was carefully pipetted out and transferred to fresh tubes. While collecting the supernatant, at least $200 \mu \mathrm{L}$ of sample was left behind in the tubes to 
prevent sampling of centrifuged fibrils at the bottom of the tube. The filtration and centrifugation steps were performed to remove any fibrils that may be present in the samples. The supernatants of both the samples were quantitated using UV spectroscopy. To maintain equal concentration of glucagon in both the samples, the glucagon trimer supernatant was diluted to $59.76 \mu \mathrm{M}$ and the glucagon control supernatant was diluted to $179.3 \mu \mathrm{M}$.

\subsubsection{Thioflavin T assay}

A $1.25 \mathrm{mM}$ thioflavin T solution was prepared by adding $12 \mathrm{ml}$ of $10 \mathrm{mM}$ PBS to 4.78 $\mathrm{mg}$ of thioflavin $\mathrm{T}$. The solution was incubated at $55{ }^{\circ} \mathrm{C}$ with intermittent shaking to dissolve the dye. The solution was allowed to come to room temperature and then filtered using a $0.22 \mu \mathrm{m}$ filter. $10 \mu \mathrm{l}$ of the $1.25 \mathrm{mM}$ ThT solution and $240 \mu \mathrm{l}$ of the glucagon samples were added into a clear bottom, black 96 well plates. Analysis was performed in triplicates. Thioflavin T measurements were taken every 15 minutes after a 5 second shaking period before each measurement. Measurements were taken using a Spectramax M5e multimode microplate reader with an excitation and emission wavelengths of $440 \mathrm{~nm}$ and $570 \mathrm{~nm}$ respectively. The concentration of glucagon trimer and glucagon control in the assay was $57.4 \mu \mathrm{M}$ and $172.2 \mu \mathrm{M}$ respectively. The concentration of thioflavin $\mathrm{T}$ in the assay is $50 \mu \mathrm{M}$. Fibrillation kinetics of the samples showing transition were fitted using the Boltzmann equation:

$\mathrm{a}+(\mathrm{b}-\mathrm{a}) /(1+\exp ((\mathrm{x}-\mathrm{c}) / \mathrm{d}))$

$\mathrm{a}=$ Maximum fluorescence recorded for the sample in the assay,

$\mathrm{b}=$ Minimum fluorescence recorded for the sample in the assay,

$\mathrm{c}=$ Inflection point

$\mathrm{d}=$ Time constant

2.10.3 Turbidity assay

172.2 $\mu \mathrm{M}$ glucagon control and $57.7 \mu \mathrm{M}$ glucagon trimer samples were prepared as described above. The glucagon control and glucagon trimer samples were added into transparent 96 well plates. Absorbance measurements were taken every 15 minutes at $405 \mathrm{~nm}$ and the plate was shaken for 5 seconds before each measurement. Measurements were taken using a Multimode DTX 880 microplate reader and the analysis was performed in triplicates. 


\subsubsection{Congo red assay}

Samples for analysis by Congo red were taken from the same trimer/glucagon stock prepared for the ThT and turbidity experiments. The Congo red stock was prepared as described by Nilsson et al [7]. Briefly, a $10 \mathrm{mM}$ Congo red stock was prepared in $5 \mathrm{mM}$ PBS 7.4, filtered through $0.22 \mu \mathrm{m}$ filter, aliquoted and stored at $-80{ }^{\circ} \mathrm{C}$. Before performing the assay, a Congo red aliquot was thawed to room temperature and diluted to $50 \mu \mathrm{M}$ using $5 \mathrm{mM}$ PBS 7.4. Freshly prepared $172.2 \mu \mathrm{M}$ glucagon control and $57.7 \mu \mathrm{M}$ glucagon trimer samples were used as non-aged samples. The glucagon samples from the turbidity assay at 62 hours were used to prepare aged samples in Congo red assay. The assay samples were prepared by adding $20 \mu \mathrm{l}$ of aged or nonaged glucagon samples to $1 \mathrm{ml}$ of Congo red working solution. These samples were then incubated at room temperature for one hour. The absorbance of the incubated samples was measured using UV spectroscopy at $477 \mathrm{~nm}$ and $540 \mathrm{~nm}$, and the concentration of Congo red bound to the fibrils was calculated using the formula as previously described [8]:

$\mathrm{C}_{b}=(\mathrm{A} 540 / 25295)-(\mathrm{A} 477 / 46306)$

A540 $=$ absorbance at $540 \mathrm{~nm}$

A477 $=$ absorbance at $477 \mathrm{~nm}$

\subsubsection{Particle size analysis using dynamic light scattering (DLS):}

Solutions of glucagon control $(172.2 \mu \mathrm{M})$ and glucagon trimer $(57.4 \mu \mathrm{M})$ were prepared in $10 \mathrm{mM} \mathrm{PBS}, 150 \mathrm{mM} \mathrm{NaCl}, \mathrm{pH} 2.5$ and aged as described in section 2.10.3. Non aged, and $70 \mathrm{~h}$ aged glucagon samples were diluted 10 fold with assay buffer and the particle size was determined using a Malvern Zetasizer Nano-ZS ZEN 3600. The experiment was performed in triplicate and z-average particle size and polydispersity index results are reported as mean \pm standard deviation. 


\section{ANALYTICAL RESULTS}

\subsection{HPLC analyses}

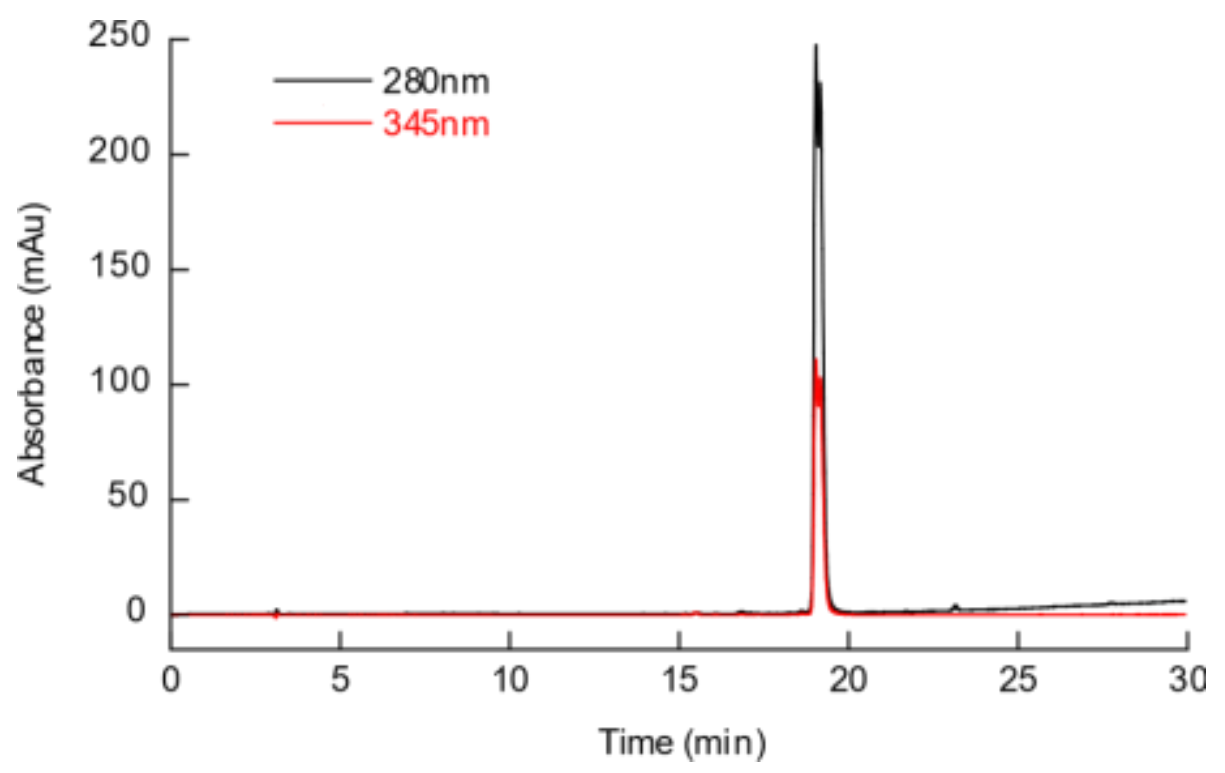

Figure S1. HPLC chromatogram of pure glucagon mono-azide

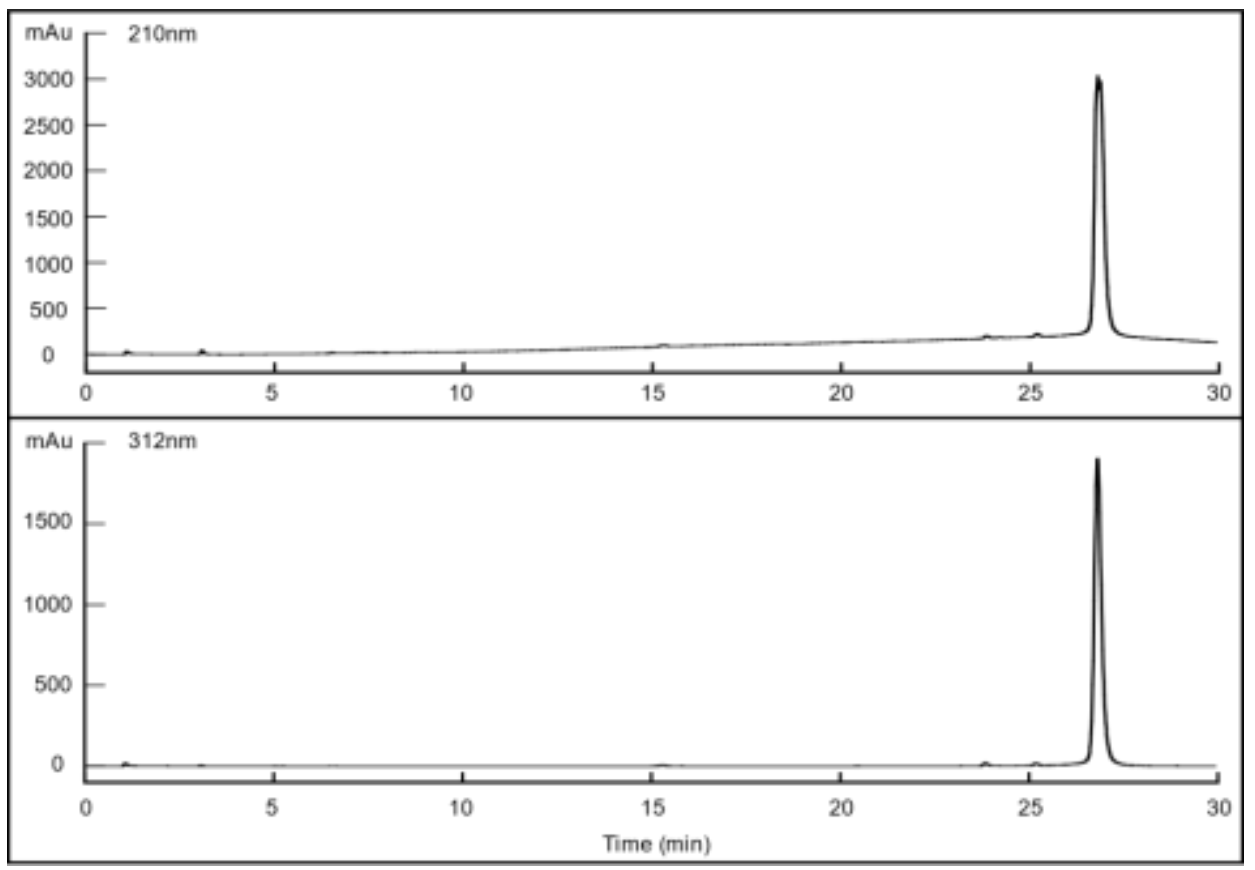

Figure S2. HPLC chromatogram of pure Tris-DBCO 


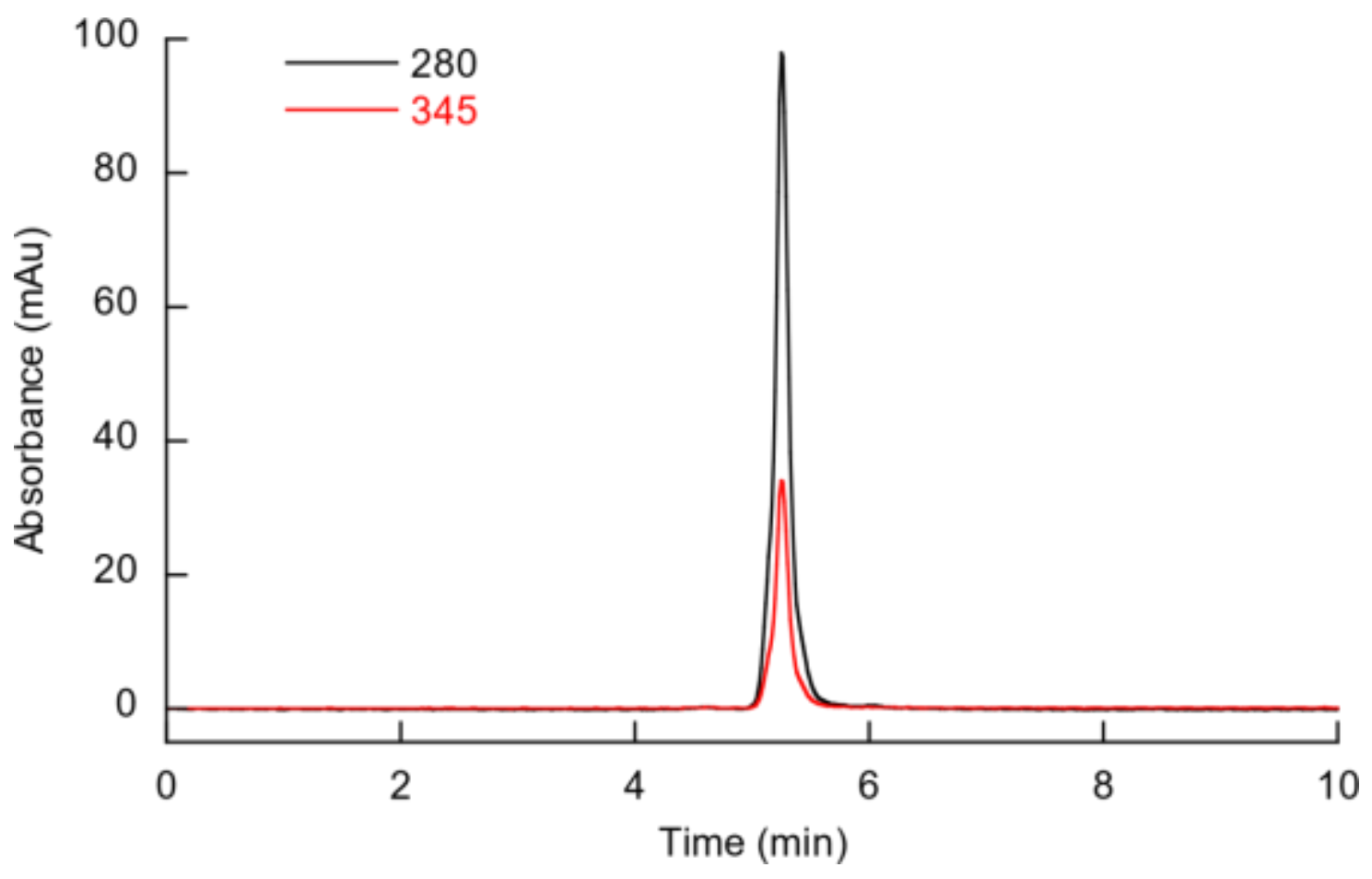

Figure S3. HPLC chromatogram of pure glucagon trimer

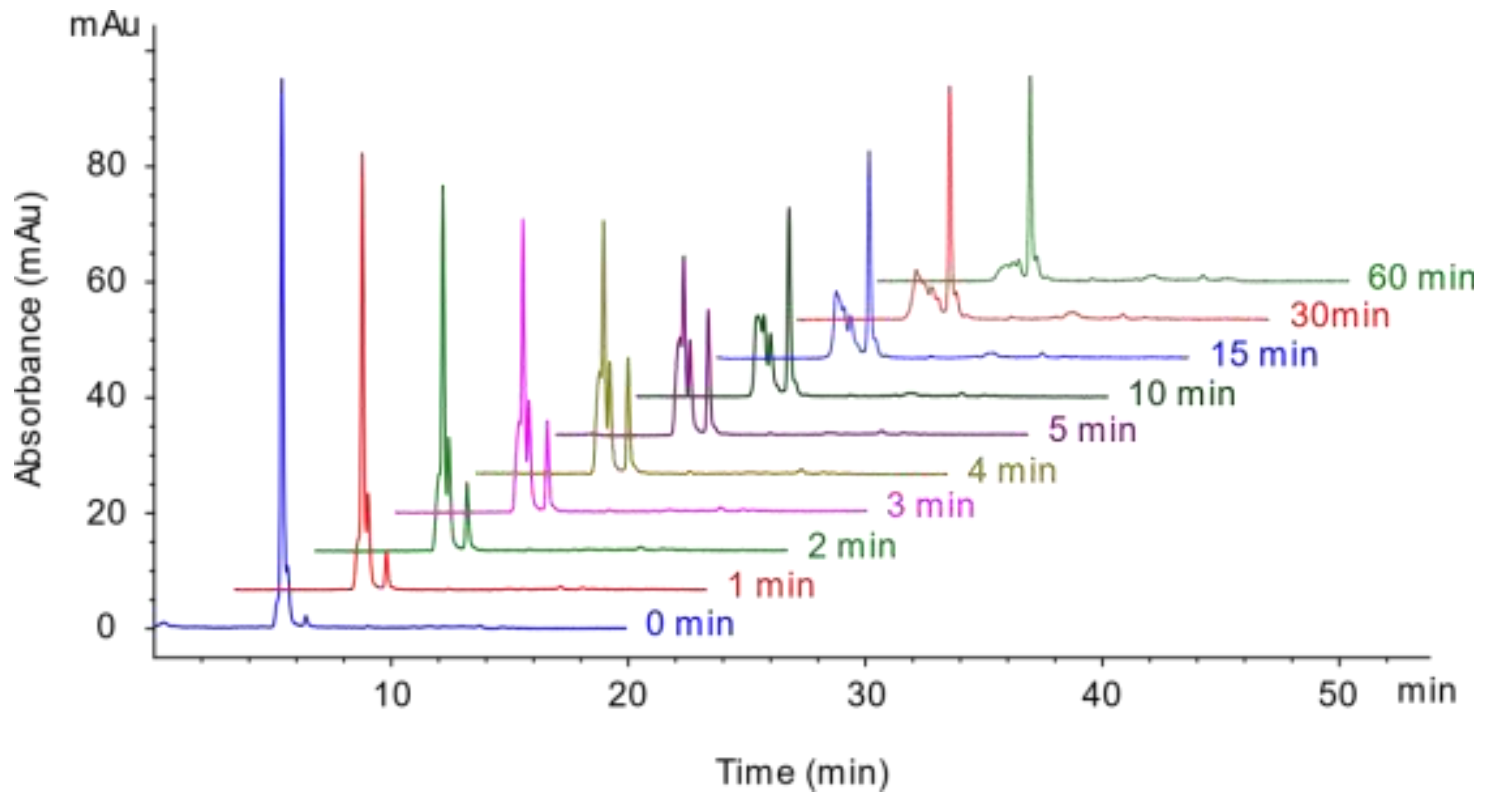

Figure S4. HPLC analysis of photolysis mixture on SEC 


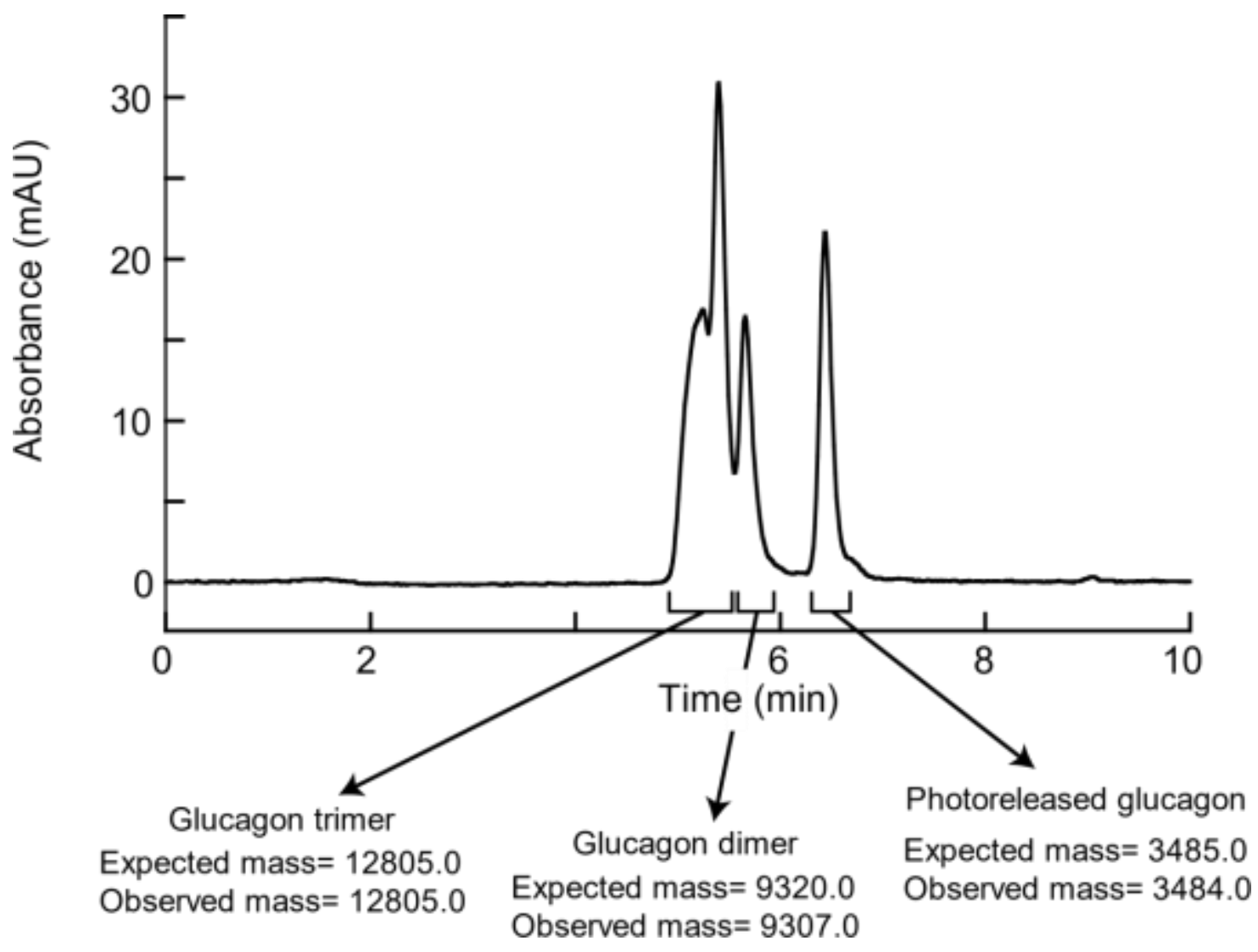

Figure S5. HPLC (size exclusion) chromatogram of photolysis reaction mixture after 5 min irradiation 


\subsection{Mass spectrometry}

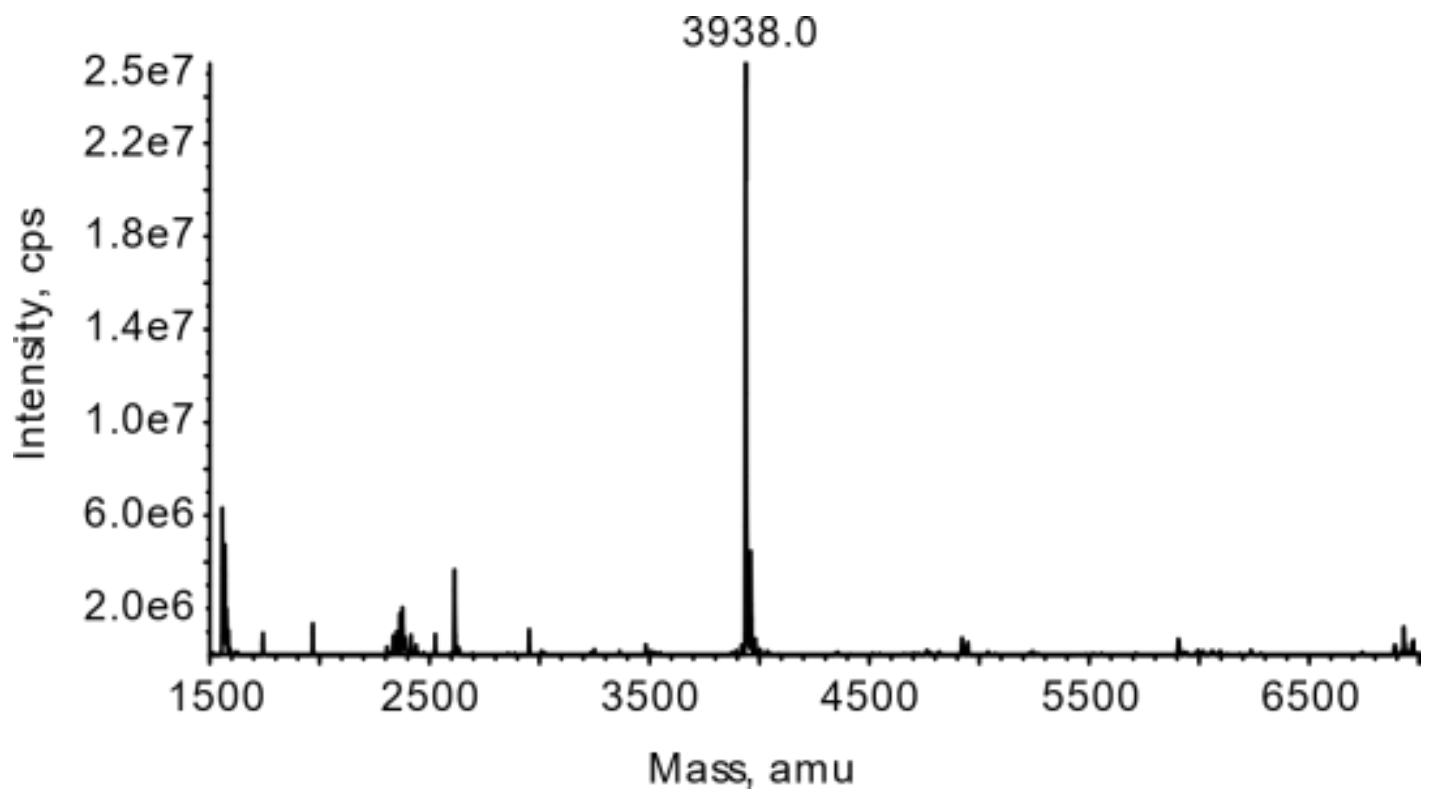

Figure S6. ESI-MS of purified glucagon mono-azide; Calculated mass: 3938.2; Observed deconvoluted mass: 3938.0

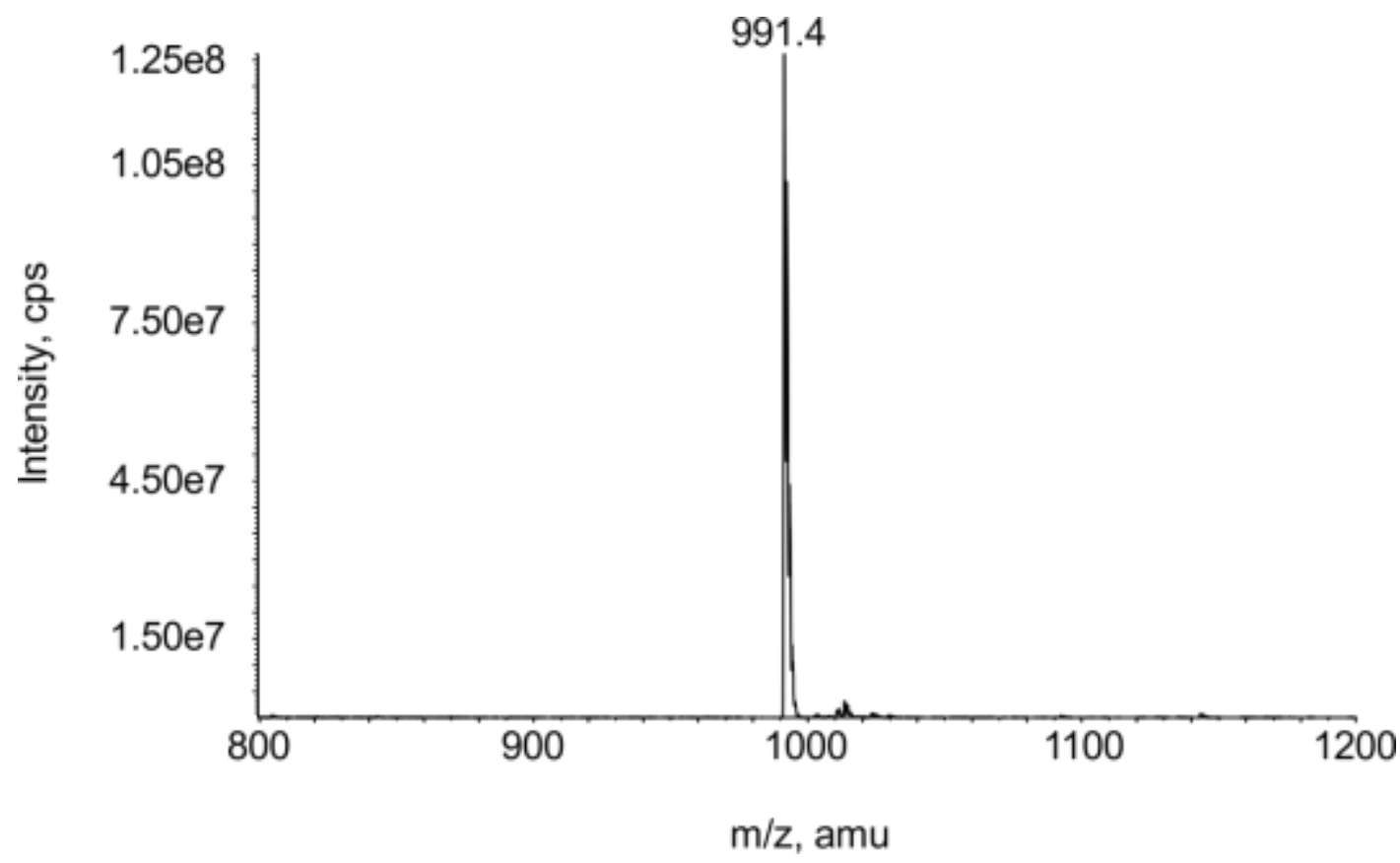

Figure S7. ESI-MS of purified Tris-DBCO; Calculated mass: $990.4 \mathrm{amu}$; Observed mass: $991.4 \mathrm{amu}$ 


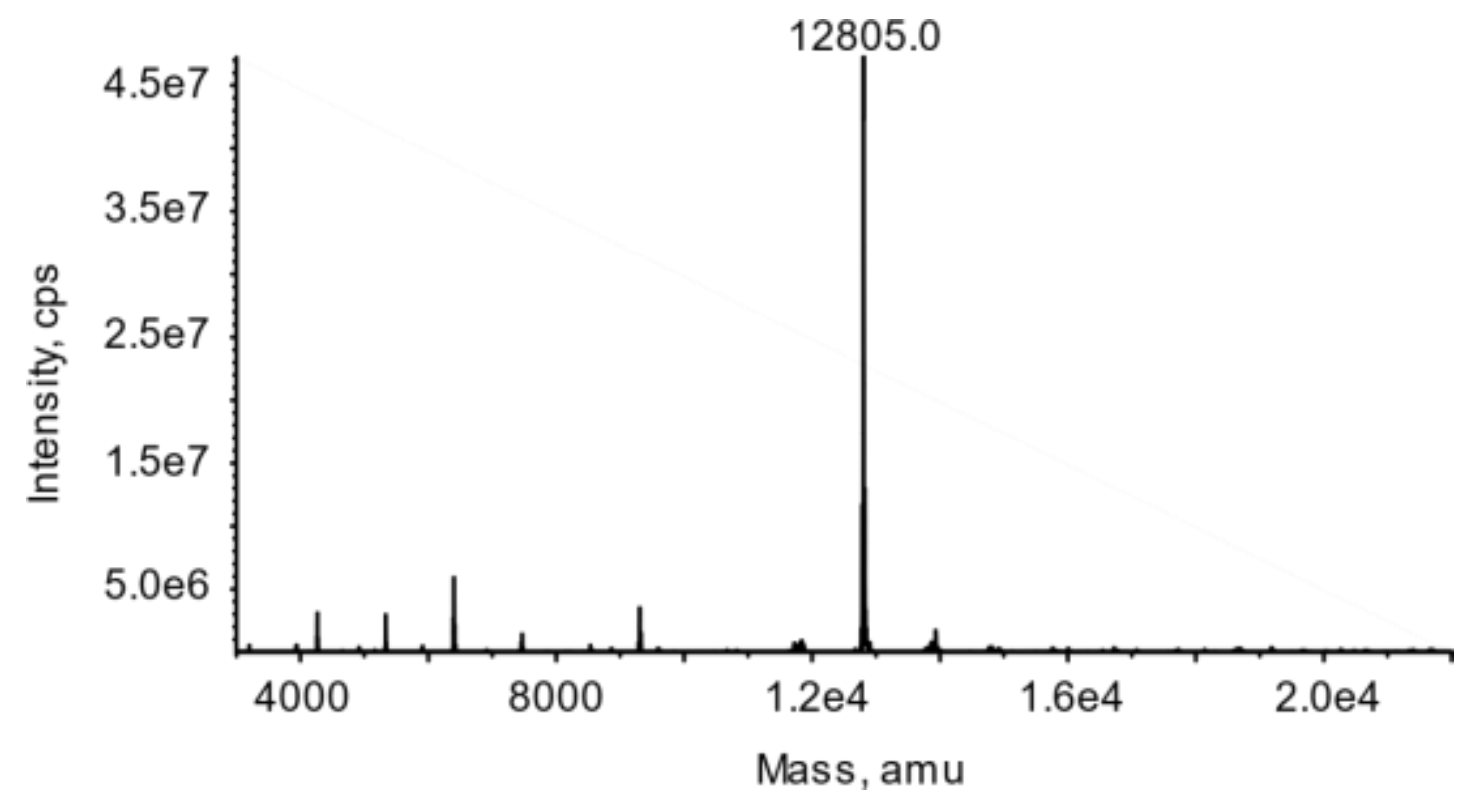

Figure S8. ESI-MS of glucagon trimer; Calculated mass: 12805.0; Observed deconvoluted mass: 12805.0

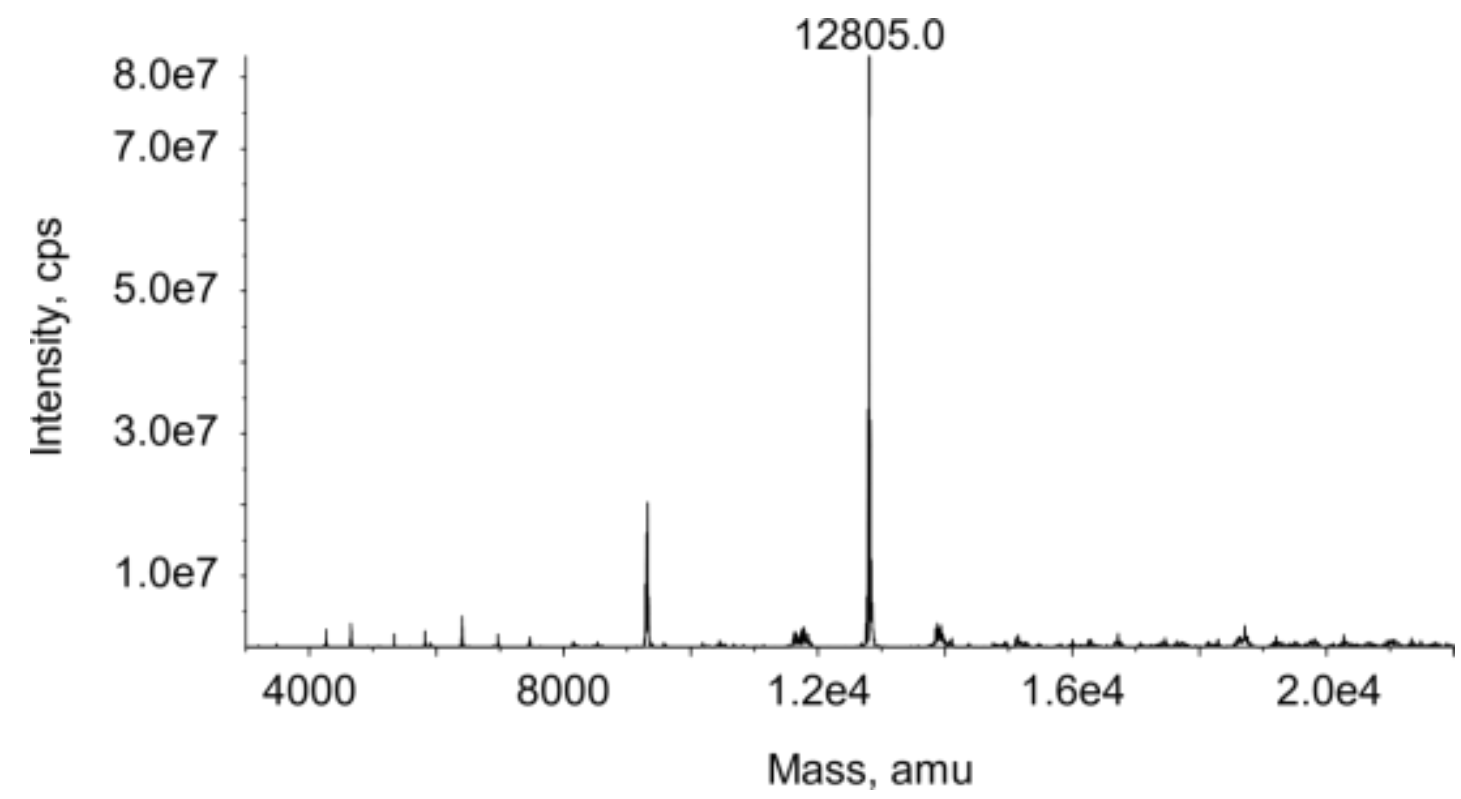

Figure S9. ESI-MS of glucagon trimer peak collected from SEC analytical run of 5 minutes irradiated photolysis mixture; Calculated mass: 12805.0; Observed deconvoluted mass: 12805.0 


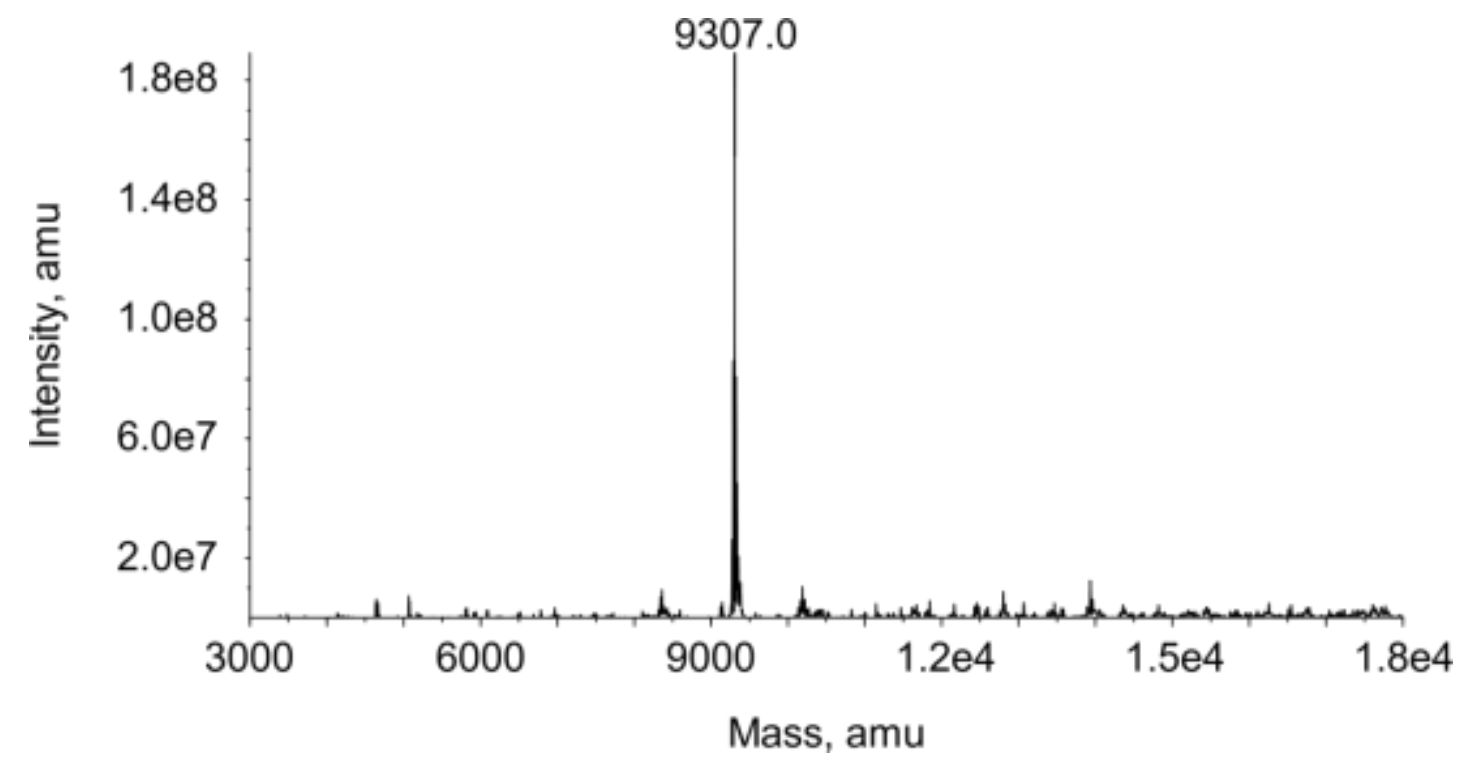

Figure S10. ESI-MS of glucagon dimer peak collected from SEC analytical run of 5 minutes irradiated photolysis mixture; Calculated mass: 9320.0; Observed deconvoluted mass: 9307.0

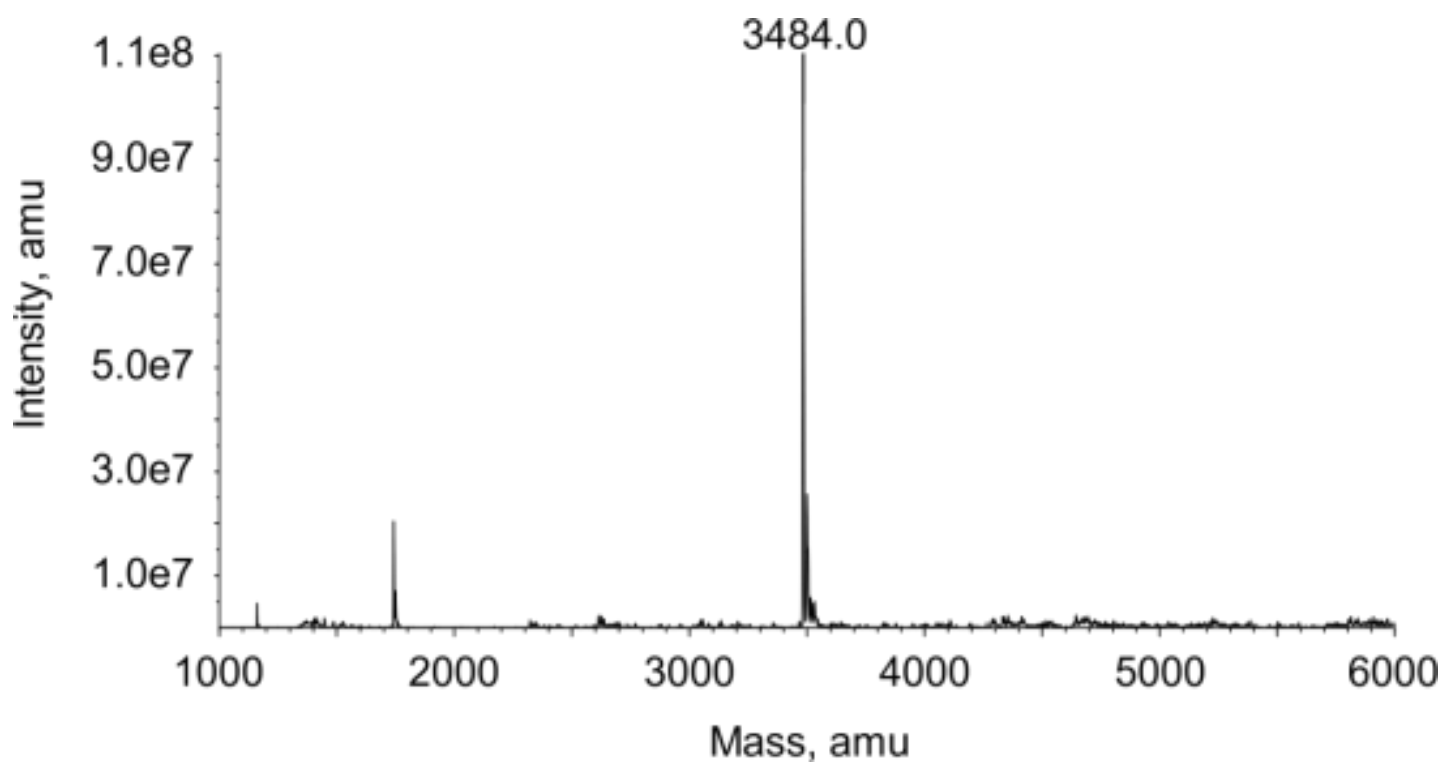

Figure S11. ESI-MS of photoreleased glucagon collected from SEC analytical run of 5 minutes irradiated photolysis mixture; Calculated mass: 3485.0; Observed deconvoluted mass: 3484.0 


\subsection{UV-visible spectroscopy}

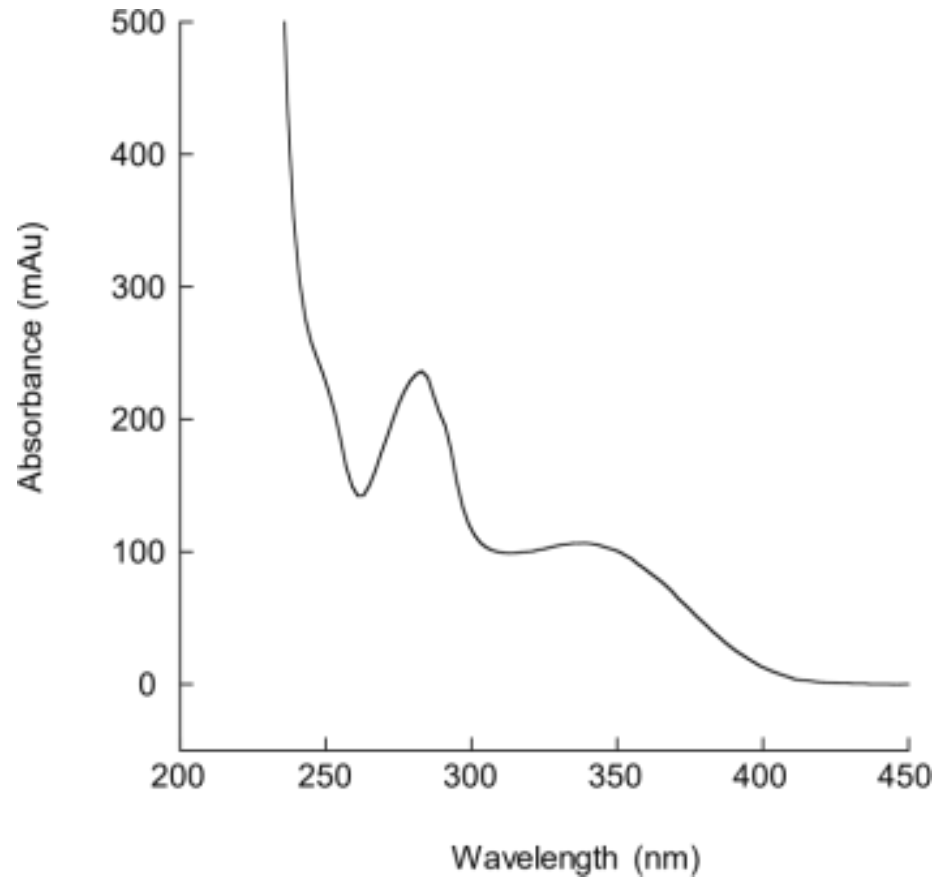

Figure S12 UV-Vis absorption spectrum of glucagon mono-azide

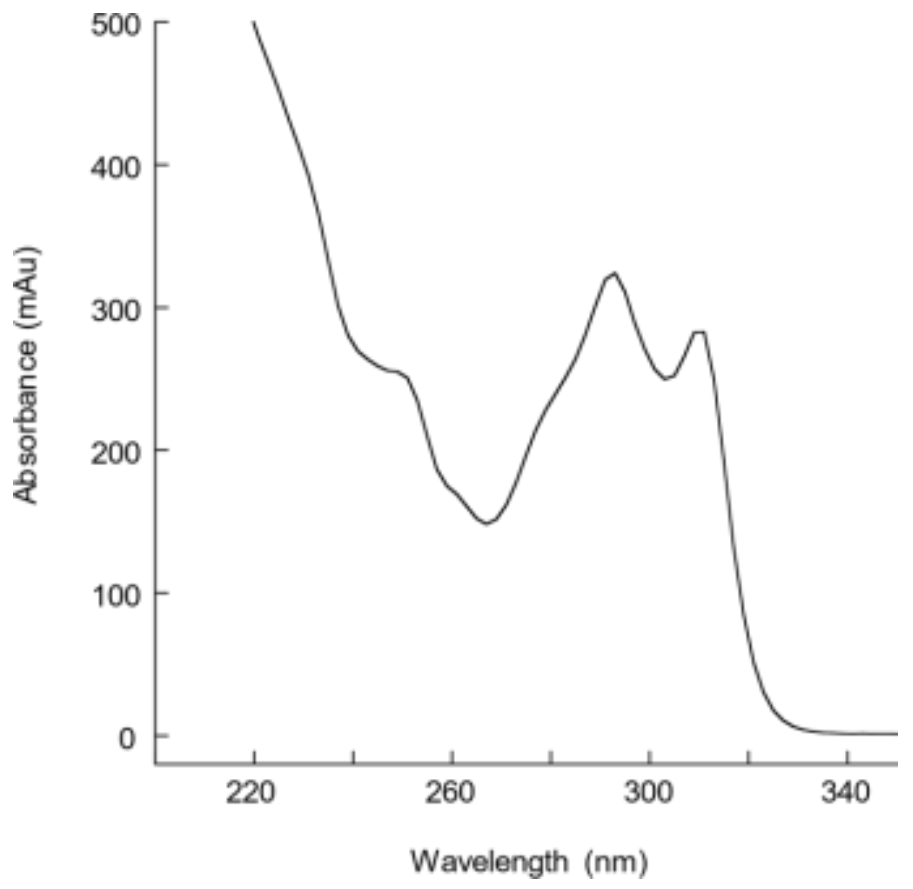

Figure S13 UV-Vis absorption spectrum of Tris-DBCO 


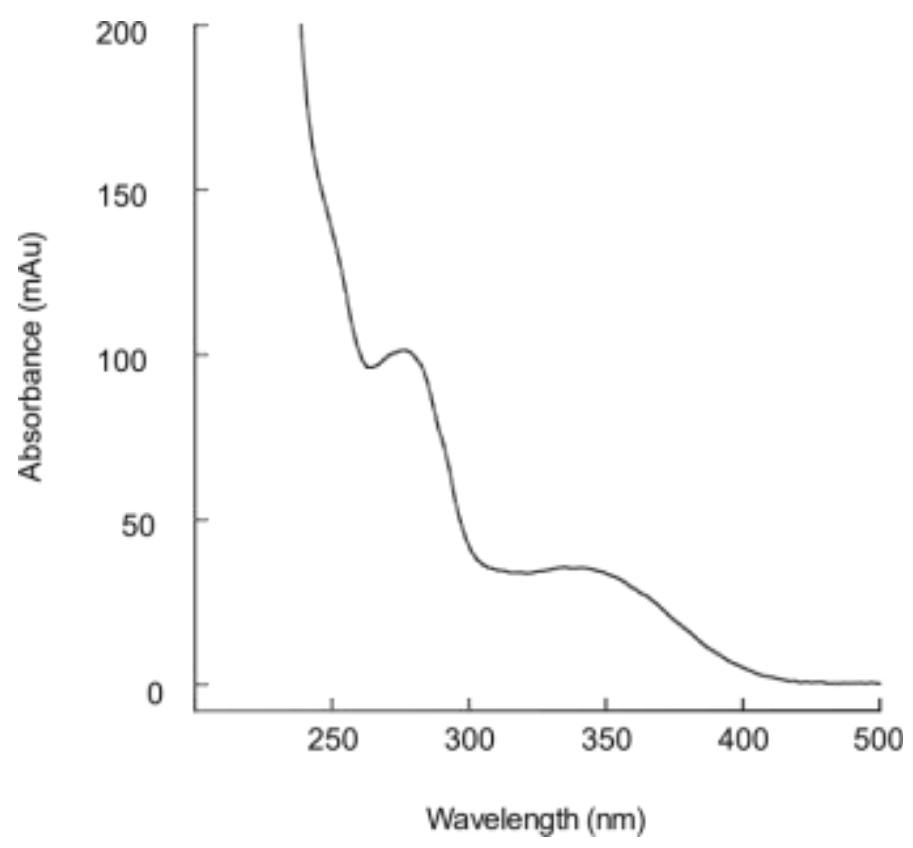

Figure S14 UV-Vis absorption spectrum of glucagon trimer 


\subsection{Isoelectric focusing of glucagon and glucagon mono-azide}

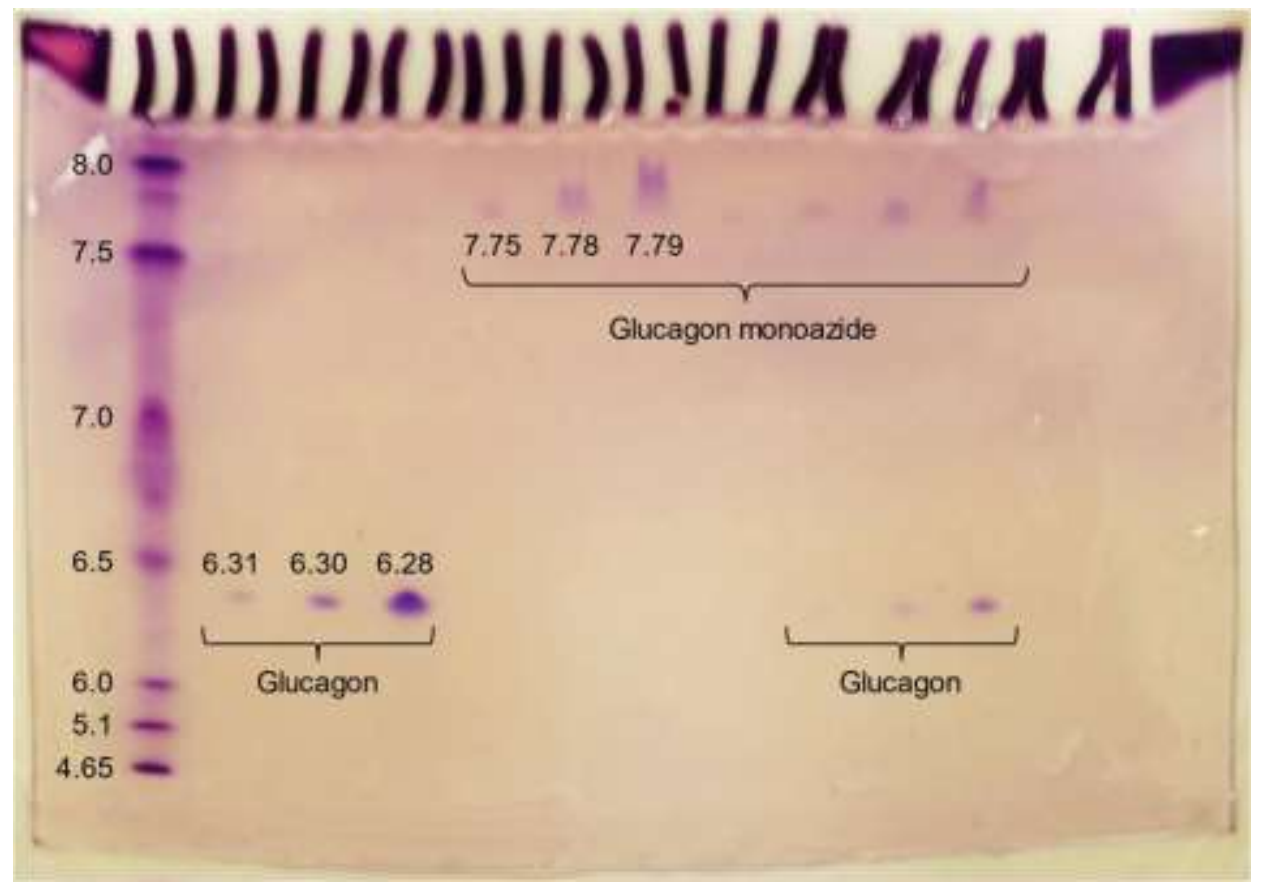

Figure S15. Isoelectric focusing of glucagon mono-azide and glucagon; Observed pI of glucagon $=6.3 \pm 0.02$; Observed $\mathrm{pI}$ of glucagon mono-azide: $7.8 \pm 0.02$

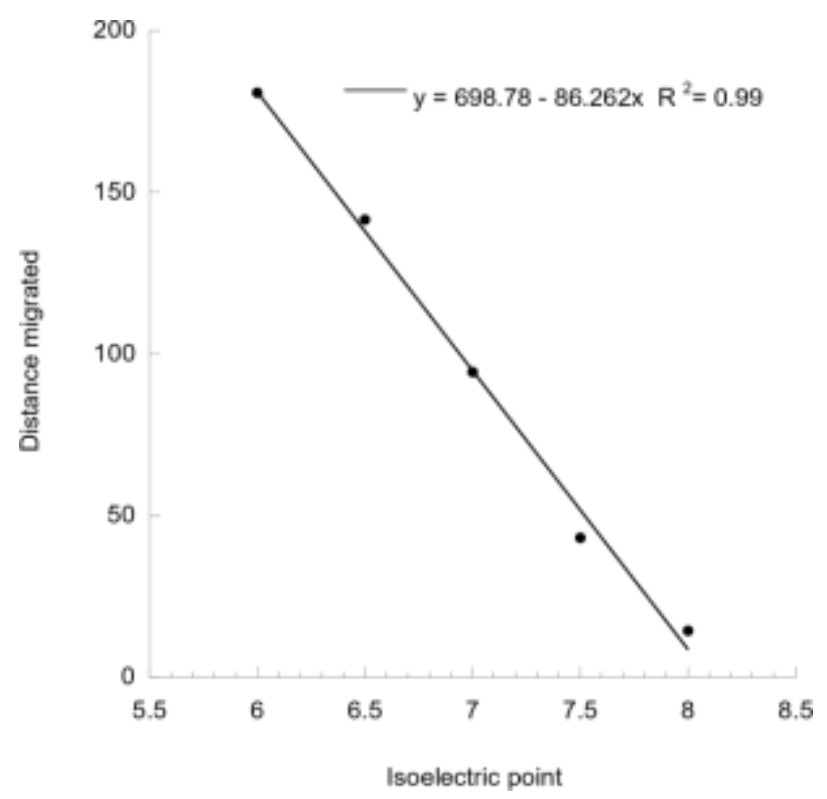

Figure S16. Standard plot used to determine the pIs of glucagon and glucagon monoazide. The plot shows the linearity of specific standards bracketing the pIs of glucagon and glucagon mono-azide 


\subsection{Glucagon trimer photolysis, supernatant analysis of centrifuged samples with extended extraction}

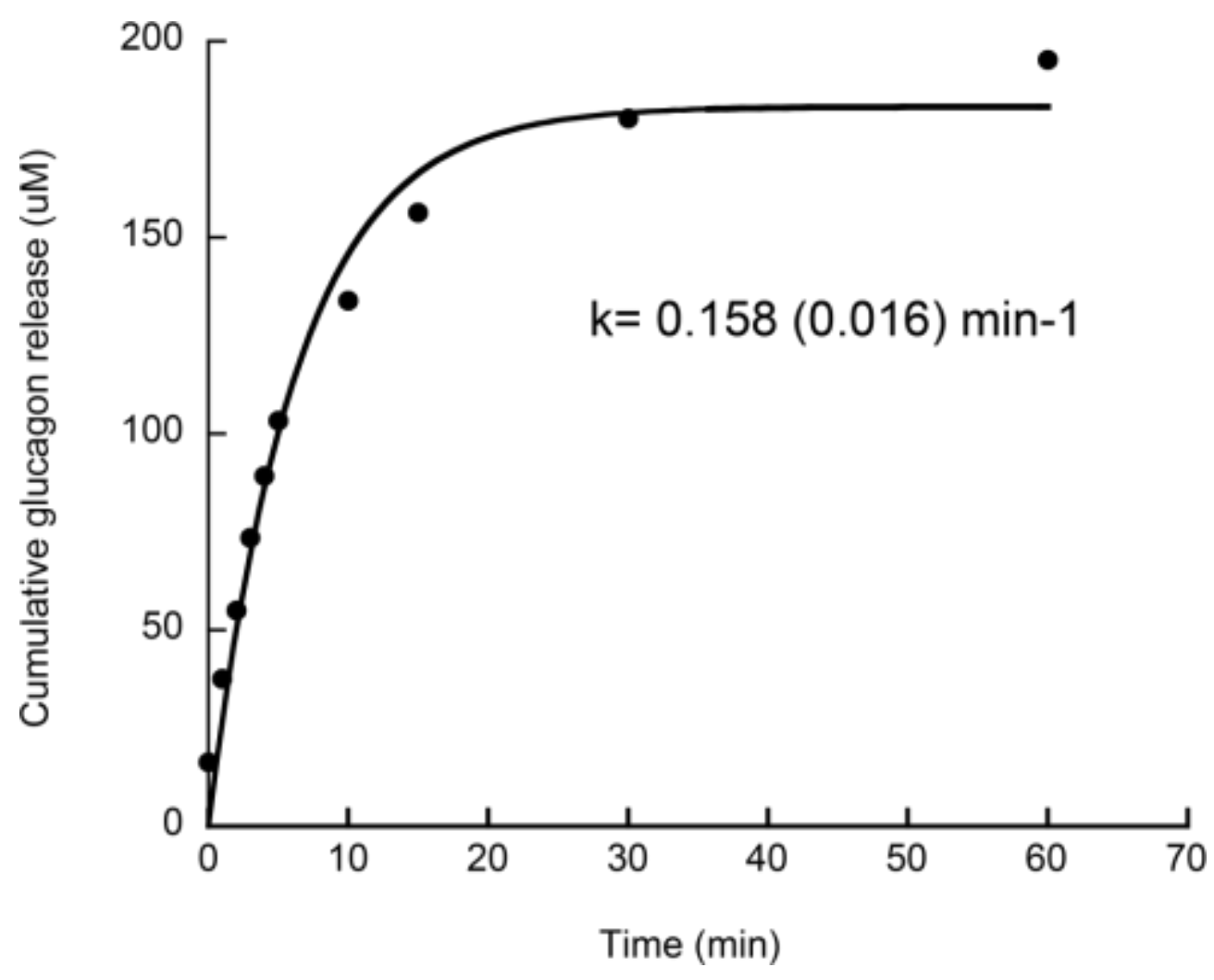

Figure S17 Photo-release of glucagon into PBS from the glucagon trimer using the extended extraction method ( 3 repetitions per time point. $100 \mu \mathrm{l}$ fresh solvent for each repetition, with 45 seconds, 5 minutes and 5 minutes vortexing respectively)

\section{REFERENCES}

[1] Jain, P.K., Karunakaran, D. and Friedman, S.H., 2013. Construction of a photoactivated insulin depot. Angewandte Chemie, 125(5), pp.1444-1449.

[2] Holmes, C.P., 1997. Model studies for new o-nitrobenzyl photolabile linkers: Substituent effects on the rates of photochemical cleavage. The Journal of Organic Chemistry, 62(8), pp.2370-2380. 
[3] Sarode, B.R., Jain, P.K. and Friedman, S.H., 2016. Polymerizing Insulin with Photocleavable Linkers to Make Light-Sensitive Macropolymer Depot Materials. Macromolecular Bioscience, 16(8), pp.1138-1146.

[4] Sarode, B. R., Kover, K., \& Friedman, S. H. (2019). Visible-Light-Activated HighDensity Materials for Controlled in Vivo Insulin Release. Molecular Pharmaceutics, 16(11), 4677-4687.

[5] Nadendla, K. and Friedman, S.H., 2017. Light control of protein solubility through isoelectric point modulation. Journal of the American Chemical Society, 139(49), pp.1786117869 .

[6] Moorthy, B.S., Ghomi, H.T., Lill, M.A. and Topp, E.M., 2015. Structural transitions and interactions in the early stages of human glucagon amyloid fibrillation. Biophysical Journal, 108(4), pp.937-948.

[7] Nilsson, M.R., 2004. Techniques to study amyloid fibril formation in vitro. Methods, 34(1), pp.151-160.

[8] Klunk, W.E., Pettegrew, J.W. and Abraham, D.J., 1989. Two simple methods for quantifying low-affinity dye-substrate binding. Journal of Histochemistry \& Cytochemistry, 37(8), pp.1293-1297. 